\title{
Frequency of KCNQ1 variants causing loss of methylation of Imprinting Centre 2 in Beckwith-Wiedemann syndrome
}

\author{
Carla Eßinger ${ }^{1}$, Stephanie Karch², Ute Moog ${ }^{3}$, György Fekete ${ }^{4}$, Anna Lengyel ${ }^{4}$, Eva Pinti ${ }^{4}$, \\ Thomas Eggermann ${ }^{1 *}$ (D) and Matthias Begemann ${ }^{1}$
}

\begin{abstract}
Background: Beckwith-Wiedemann syndrome (BWS) is an imprinting disorder caused by disturbances of the chromosomal region 11 p15.5. The most frequent molecular finding in BWS is loss of methylation (LOM) of the Imprinting Centre 2 (IC2) region on the maternal allele, which is localised in intron 10 of the KCNQ1 gene. In rare cases, LOM of IC2 has been reported in families with KCNQ1 germline variants which additionally cause long-QT syndrome (LQTS). Thus, a functional link between disrupted KCNQ1 transcripts and altered IC2 methylation has been suggested, resulting in the co-occurrence of LQTS and BWS in case of maternal inheritance. Whereas these cases were identified by chance or in patients with abnormal electrocardiograms, a systematic screen for KCNQ1 variants in IC2 LOM carriers has not yet been performed.
\end{abstract}

Results: We analysed 52 BWS patients with IC2 LOM to determine the frequency of germline variants in KCNQ1 by MLPA and an amplicon-based next generation sequencing approach. We identified one patient with a splice site variant causing premature transcription termination of KCNQ1.

Conclusions: Our study strengthens the hypothesis that proper KCNQ1 transcription is required for the establishment of IC2 methylation, but that KCNQ1 variants cause IC2 LOM only in a small number of BWS patients.

Keywords: Beckwith-Wiedemann syndrome, KCNQ1 variants, Imprinting Centre 2, Loss of methylation, Long-QT syndrome

\section{Background}

Beckwith-Wiedemann syndrome (BWS, OMIM \#130650) is a congenital imprinting disorder which was first described by Beckwith and Wiedemann in 1963 [1, 2]. Clinically, it presents with features including macroglossia, exomphalos, lateralised overgrowth, an increased risk for Wilms tumour, prolonged hyperinsulinism after birth and several other symptoms (for review: [3]). In the past, the phenotypic heterogeneity has hindered the clinical

\footnotetext{
* Correspondence: teggermann@ukaachen.de

'Institute of Human Genetics, Medical Faculty, RWTH Aachen University, Pauwelsstr. 30, 52074 Aachen, Germany

Full list of author information is available at the end of the article
}

diagnosis; therefore, in 2018, Brioude et al. have introduced a consensus scoring system that allows the classification of patients with BWS according to the symptoms and the molecular alteration [3].

BWS is associated with disturbances of the chromosomal region 11p15.5 which harbours two imprinting control regions [4]. The Imprinting Centre 1 (IC1; H19/ IGF2:IG-DMR) is paternally methylated and regulates the monoallelic expression of the insulin-like growth factor 2 (IGF2) and H19 genes. The Imprinting Centre 2 (IC2; KCNQ1OT1:TSS-DMR) controls the expression of the genes KCNQ1, KCNQ1OT1 and CDKN1C. In contrast to the IC1, the IC2 is maternally methylated leading

(c) The Author(s). 2020 Open Access This article is licensed under a Creative Commons Attribution 4.0 International License, which permits use, sharing, adaptation, distribution and reproduction in any medium or format, as long as you give appropriate credit to the original author(s) and the source, provide a link to the Creative Commons licence, and indicate if changes were made. The images or other third party material in this article are included in the article's Creative Commons licence, unless indicated otherwise in a credit line to the material. If material is not included in the article's Creative Commons licence and your intended use is not permitted by statutory regulation or exceeds the permitted use, you will need to obtain permission directly from the copyright holder. To view a copy of this licence, visit http://creativecommons.org/licenses/by/4.0/. The Creative Commons Public Domain Dedication waiver (http://creativecommons.org/publicdomain/zero/1.0/) applies to the data made available in this article, unless otherwise stated in a credit line to the data. 
to an expression of $K C N Q 1$ and $C D K N 1 C$ and a repression of KCNQ1OT1 on the maternal allele, while it is unmethylated on the paternal allele resulting in silencing of KCNQ1 and CDKN1C and expression of KCNQ1OT1 (for review: [5]).

The most frequent molecular cause of BWS is loss of methylation (LOM) of the IC2 (50-60\% of patients) causing a biallelic expression of $K C N Q 1 O T 1$ and repression of maternally expressed genes [4]. Further alterations comprise a paternal uniparental disomy (UPD) of chromosome 11p15.5 (20-25\%) and a gain of methylation (GOM) at the IC1 $(\sim 4 \%)$ (for review: [3]). Point mutations of CDKN1C are rare in sporadic BWS (1.3-5\%) but more common in familial cases of BWS (20-40\%) [6]. Other molecular cases such as copy number variants (CNVs) of the IC1 and/or IC2 only occur in single cases $(<1 \%)$ [7]. An epigenotype-phenotype correlation could be delineated for some symptoms, such as omphalocele, hemihypertrophy and the risk to develop neoplasias [8].

BWS mostly occurs sporadically, but in addition to inherited variants in CDKN1C [9], familial occurrence has also been described for chromosomal rearrangements and CNVs in IC1 and IC2 [6], among them deletions within the KCNQ1 gene [10-13]. Some of them include the IC2 domain itself and are therefore associated with a LOM of the IC2. However, there are also cases where a loss of transcription of $K C N Q 1$, either by CNVs or single nucleotide variants (SNVs), causes a LOM of the IC2 [14-16]. Remarkably, these genetic alterations affect only KCNQ1 and its regulatory elements, but not the IC2 itself. In mice, a maternally inherited poly(A) cassette, inserted into Kcnq1, terminates the transcription of Kcnq1 upstream of IC2 and results in LOM of the IC2, biallelic expression of Kcnq1ot1 and silencing of Cdkn1c and Kcnq1 [17].

Loss-of-function mutations in KCNQ1 are the most frequent causes for congenital long-QT syndrome (LQTS) [18], a hereditary cardiac disease with a prolonged QT interval resulting in a significant risk of arrhythmias and sudden death (for review: [19]). The penetrance of LQTS is variable as only about $60 \%$ of carriers of a pathogenic KCNQ1 variant have a prolonged QT interval. Vice versa, $10 \%$ of the genetically affected patients who do not have a prolonged QT interval can be affected by a cardiac event until the age of 40 years [20]. It is conceivable that apparently sporadic BWS cases with IC2 LOM are caused by familial pathogenic KCNQ1 variants but remained undetected so far due to the reduced penetrance of LQTS variants. Up to now, six LQTS families have been described in which altered KCNQ1 transcripts lead to BWS in combination with LQTS when inherited from the mother or to isolated LQTS when transmitted from the father [14, 21-23]. The different consequences depend on the parental origin of the allele carrying the altered $K C N Q 1$ variant and can therefore be explained by the biallelic expression of KCNQ1 in the heart but monoallelic expression in other tissues [21]. However, these were mainly case reports and a systematic screen for $K C N Q 1$ variants in BWS patients associated with IC2 LOM is missing.

In our study, we therefore aimed to determine the frequency of pathogenic germline variants in KCNQ1 among BWS patients with IC2 LOM.

\section{Results}

Copy number analysis of the coding sequence of KCNQ1 by multiplex ligation-dependent probe amplification (MLPA) did not reveal any deletion or duplication in the cohort of 52 BWS patients with IC2 LOM.

By an amplicon-based next generation sequencing (NGS) approach, heterozygosity for a splice variant in KCNQ1 was detected in one patient. The variant was located in the donor splice site of intron 1 of KCNQ1 (NM_000218.2: c.386+1G >T; Chr11:2466715G>T, HG19). The change was also detected in the mother but not in the father. The variant affects the canonical donor splice site of intron 1 (MaxEnt, NNSplice, SSF, - 100\%) (Fig. 1) and is therefore predicted to impair the transcription of KCNQ1.

\section{Patient with IC2 LOM and KCNQ1 variant}

The boy was born at 26 weeks of gestation by caesarean section due to premature placental separation and preeclampsia with a birthweight of $950 \mathrm{~g}$ (50th percentile), a length of $33 \mathrm{~cm}$ (30th percentile) and a head circumference of $23 \mathrm{~cm}$ (15th percentile). He showed marked macroglossia, cleft palate, umbilical and inguinal hernias, hypospadias, ear lobe creases and a nevus flammeus in his face. He suffered from chronic respiratory insufficiency due to bronchopulmonary dysplasia as a consequence of his prematurity. He underwent multiple surgeries during the first months of life, including reduction of macroglossia. He showed a severe feeding disorder and required tube feeding for several years. His psychomotor development was significantly delayed putatively due to his premature birth. At the age of 3 years, he was able to walk without support, and he started speaking a few words at the age of 7 years. On the last examination at the age of eight and a half years, he was attending a school for special needs. His vision was restricted because of retinopathy of prematurity, and he needed regular controls for a nephrocalcinosis ${ }^{\circ} \mathrm{I}-\mathrm{II}$.

Clinical diagnosis of BWS was confirmed by molecular detection of IC2 LOM at the age of 2 months. Molecular karyotyping did not reveal any CNVs (resolution of 50 $\mathrm{kb})$. He had one healthy brother. His mother was healthy as well and did not show any BWS features. MLPA of the mother showed a normal methylation pattern.

An electrocardiography (ECG) of the patient was performed after the results of the molecular analysis of KCNQ1 and revealed a borderline prolonged QTc 


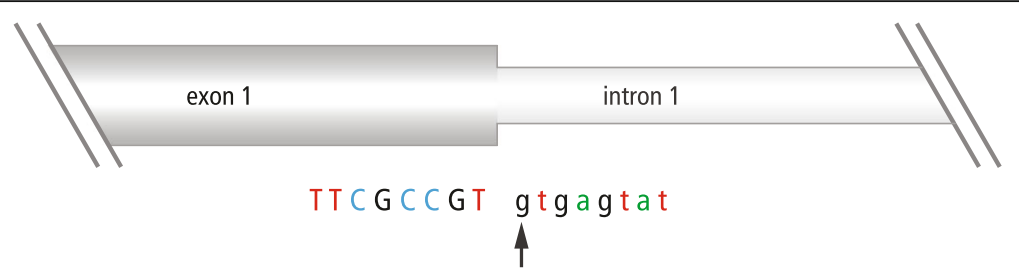

NM_000218.2(KCNQ1):C.386+1G>T
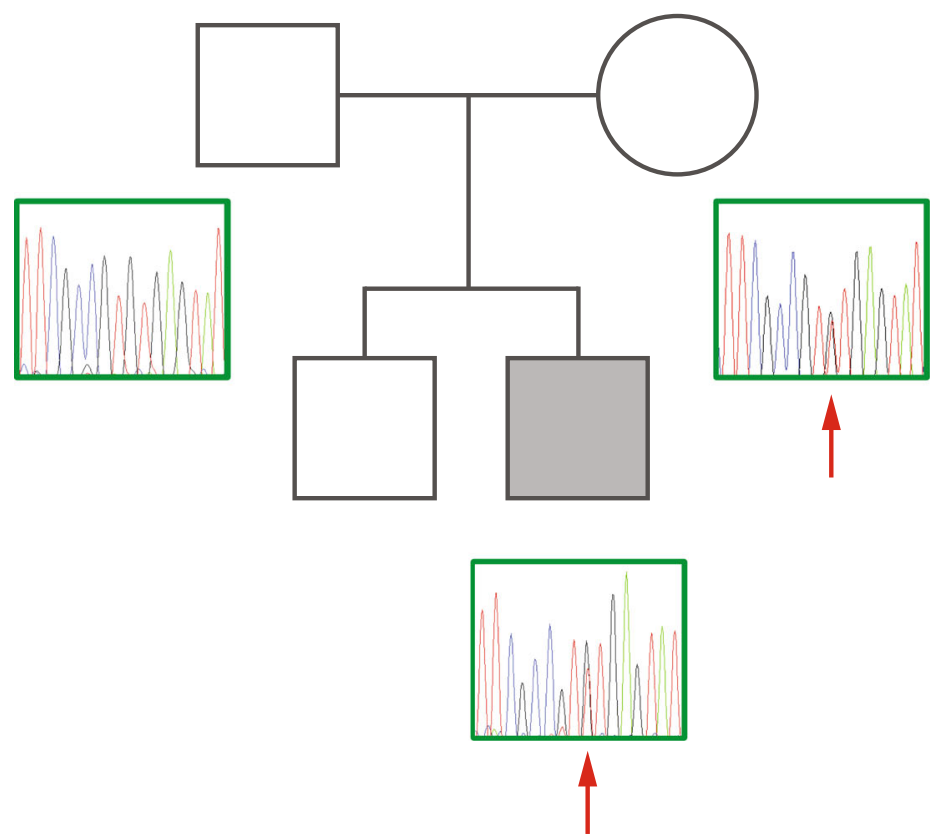

Fig. 1 Sequencing results of the family with the donor splice site variant in intron 1 of the KCNQ1 gene. The patient and the patient's mother carry the splice site variant in intron 1 of KCNQ1 (NM_000218.2(KCNQ1):c.386+1G>T). The patient is diagnosed with BWS due to IC2 LOM. The mother and the brother of the patient are healthy

interval of 440-450 ms. The long-term ECG showed normal findings but sporadic extrasystoles. The mother's ECG revealed no pathologic findings.

\section{Discussion}

By analysing 52 BWS patients with IC2 LOM for $K C N Q 1$ germline variants, we identified one case with a maternally inherited splice site variant $(c .386+1 \mathrm{G}>\mathrm{T})$ affecting the splice donor site of intron 1 of KCNQ1. A variant at the same position with another base pair substitution $(\mathrm{c} .386+1 \mathrm{G}>\mathrm{C})$ has been described by Valente et al. [21] in a BWS patient with IC2 LOM (patient 2). This patient showed typical BWS features and was diagnosed with LQTS on the basis of an ECG performed after an episode of chest pain. The patient's mother did not show any signs of BWS but was diagnosed as asymptomatic LQTS with a prolonged QT interval [21]. The authors suggested that the splice site variant leads to a premature transcription termination and a disturbed elongation of the KCNQ1 transcript. The resulting monoallelic expression of KCNQ1 could explain the LQTS phenotype as KCNQ1 is expressed biallelically in the heart [21]. In tissues with monoallelic expression of the maternal allele, the altered transcript should cause a LOM of the IC2 (Fig. 2).

Five further cases corroborate the hypothesis that interrupted transcription of $K C N Q 1$ upstream of IC2 results in LOM. A deletion in KCNQ1 has been reported that does not include the IC2 but causes LOM, suggesting that transcription of the entire KCNQ1 gene is required for the establishment of the imprinting mark at the IC2 locus [14]. Demars et al. described a 50-kb duplication in KCNQ1 upstream of IC2 which leads to an IC2 LOM and BWS symptoms when inherited from the mother but to a normal phenotype in case of paternal inheritance [15]. In a third family, a translocation with a breakpoint in intron 9 of KCNQ1 results in a LOM of the IC2 and BWS when maternally inherited [16]. 


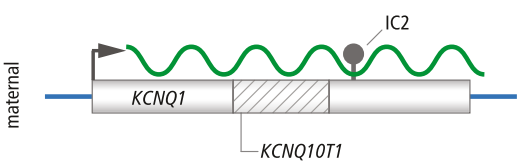

situation in

healthy individual
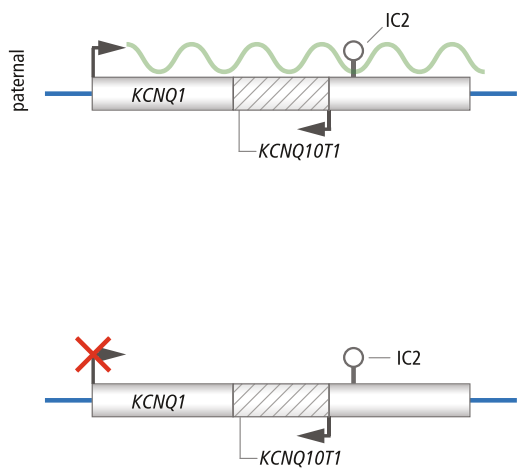

clinical variant

$\rightarrow$ premature transcription termination of KCNQ1

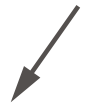

maternal transmission
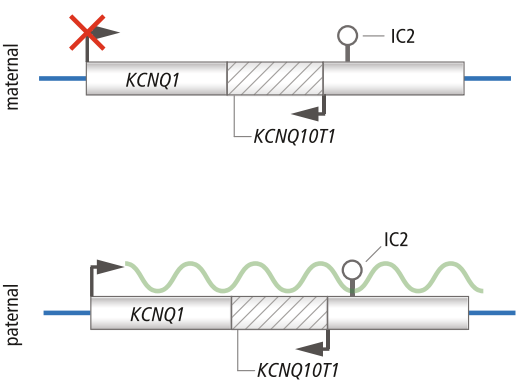

BWS + LQTS
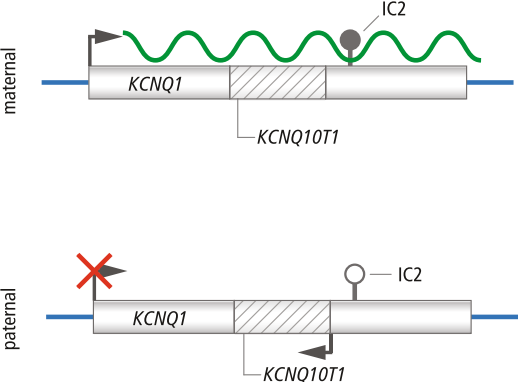

LQTS

Fig. 2 Hypothesised model of the effect of premature transcription termination of KCNQ1 on the methylation status of the IC2 (figure modified from Valente et al. [21]). In healthy individuals, IC2 is methylated on the maternal allele (indicated by a filled lollipop) leading to an expression of KCNQ1 and a repression of KCNQ1OT1. On the unmethylated paternal allele (indicated by an open lollipop), KCNQ1 is repressed but KCNQ1OT1 is expressed. Active promoters and transcription are indicated by bent black arrows, and correct transcription of KCNQ1 is depicted by a Curved dark green line. The curved light green line indicates the paternal transcription of KCNQ1 in the heart. Due to a variant in KCNQ1, the transcription of KCNQ1 is disturbed (indicated by a red cross). After maternal transmission, the variant leads to BWS due to the LOM of IC2 and, due to haploinsufficiency of KCNQ1, to LQTS. After paternal transmission, the variant may lead to isolated LQTS due to the haploinsufficiency of KCNQ1

Valente et al. described two additional patients: one with a deletion in exon 1 of KCNQ1 and one with a 160-kb KCNQ1 duplication. Both patients were affected by LQTS and BWS due to an IC2 LOM [21]. In our case, LQTS could not be detected in the patient's mother but this finding can be explained by the variable penetrance of LQTS [20]. The borderline prolonged QTc interval associated with the probably pathogenic variant found in the patient makes LQTS in the patient very likely.
In fact, functional analyses have not been conducted but the effect of the splice site variant can be delineated from findings published recently by Valente et al. [21].

Up to now, only case reports and CNV studies have been published, but systematic studies to determine the frequency of KCNQ1 variants in BWS patients with IC2 LOM have not yet been performed. Demars et al. screened 78 BWS patients with IC2 LOM for CNVs and discovered one patient with a duplication 
including exon 2 and parts of intron 1 and 2 of KCNQ1. However, they did not perform ECG examinations [15]. Similarly, Baskin et al. discovered two out of 103 BWS patients with CNVs within the IC2 region but ECG data were not provided [13]. Valente et al. restricted their mutation analysis to three BWS patients with ECG abnormalities and therefore might have missed KCNQ1 mutation carriers without a QT prolongation [21]. In addition to these studies, single case reports on BWS families with KCNQ1 germline variants and multiple miscarriages $[14,16]$ or episodes of cardiac arrhythmia [22, 23] have been published. Other case reports describe further deletions in KCNQ1 [10-12]. Thus, this is the first systematic screening of a cohort of BWS patients with IC2 LOM for pathogenic variants in the KCNQ1 gene. However, our screening report has some technical limitations. The used MLPA kit does not cover exon 5 of KCNQ1 (see supplementary table); furthermore, deletions outside of the MLPA probe's hybridisation sites might escape detection. The amplicon-based NGS approach only identifies exonic variants while variants in non-coding regions may not be covered by the primers (see supplementary table).

Altogether, only a small number of patients with both functional relevant $K C N Q 1$ variants and IC2 LOM have been identified; therefore, the frequency of BWS patients at risk for KCNQ1-related LQTS appears to be low. However, it can be assumed that the frequency of KCNQ1 germline variants in BWS might be higher due to incomplete penetrance of $K C N Q 1$ variants. In fact, about $40 \%$ of carriers of pathogenic $K C N Q 1$ variants do not exhibit a prolonged QT interval [20]. However, it is important to identify patients with a KCNQ1 variant that may lead to LQTS, as these individuals are at risk of developing cardiac events even when asymptomatic. This also applies to relatives of these patients in case they are carriers of the variant. Furthermore, cardiac events can also occur in genetically affected persons without QT prolongation [20].
The identification of $K C N Q 1$ variants leading to an altered transcription of KCNQ1 is relevant not only for the prediction of LQTS but also for the recurrence risk of BWS. The majority of BWS cases occur sporadically, but in case of IC2 LOM caused by a germline variant in KCNQ1, the probability of transmission and the risk of offspring suffering from BWS and LQTS are increased [13]. The risk of transmission via the maternal germline and therefore to suffer from BWS is $50 \%$, whereas it is generally $50 \%$ for LQTS regardless of the parental origin of the allele.

\section{Conclusions}

The correlation between BWS and LQTS has an impact on the clinical recommendations and surveillance of the patients with both diseases. Early diagnosis of LQTS is important to prevent life-threatening cardiac arrhythmias. The first line therapy of LQTS is a treatment with $\beta$ blockers (e.g. propranolol). In case of therapy refractoriness, left cardiac sympathetic denervation or an implantable cardioverter defibrillator (ICD) is necessary. Also, the patients should avoid competitive sports (e.g. swimming) and the intake of QT interval prolonging drugs (for review: [19]). The precise molecular diagnosis of BWS is important for the clinical management, the screening protocol and the estimation of the recurrence risk. An international consensus group has already recommended to perform an ECG in patients with CNVs involving the IC2 region [3]. However, screening for KCNQ1 variants is not carried out routinely and SNVs may escape routine diagnostic detection. As screening for KCNQ1 variants in all IC2 LOM patients would be expensive, an ECG in this group of patients is a suitable examination tool and should be performed in every patient with IC2 LOM.

\section{Methods}

\section{Patients}

We investigated 52 BWS patients carrying an IC2 LOM. The molecular diagnosis had been confirmed by

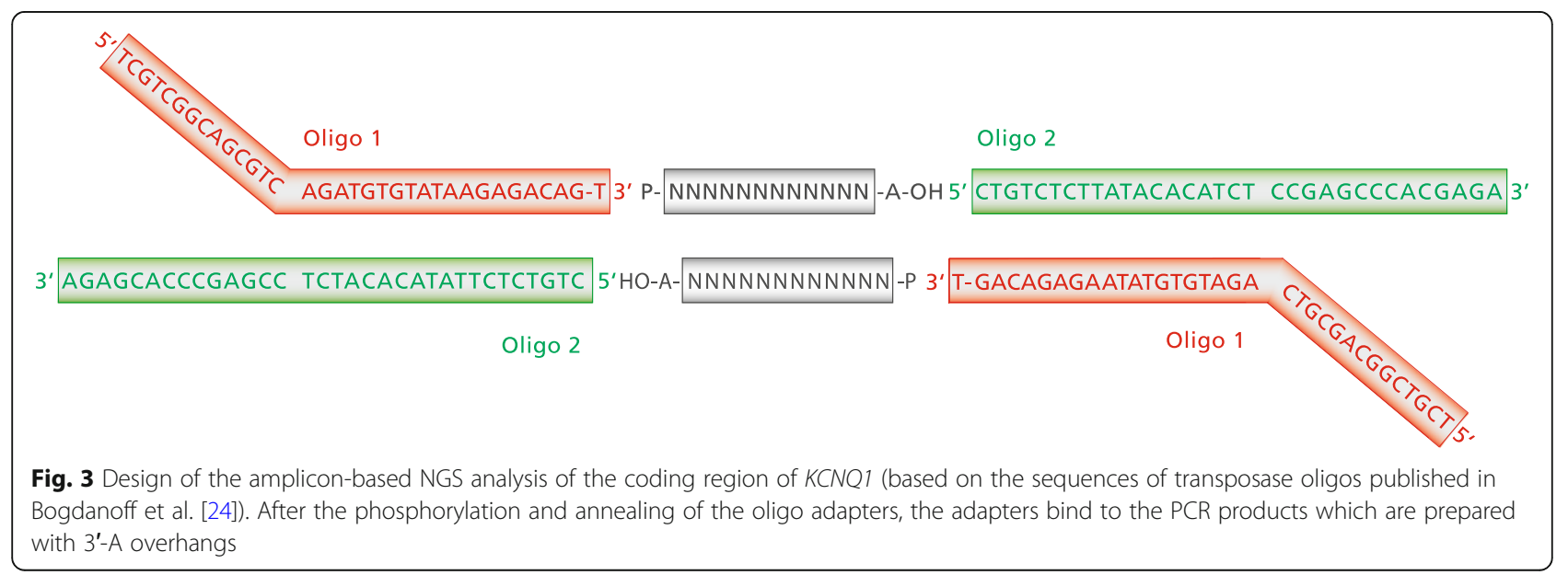


methylation-specific multiplex ligation-dependent probe amplification (MS-MLPA; assay ME030, MRC-Holland, Amsterdam, The Netherlands) according to the manufacturer's instructions. Multilocus imprinting disturbances (MLID) were excluded by screening with a MSMLPA-based multilocus assay (assay ME034, MRCHolland). Patients with MLID were excluded as they are probably caused by other (genomic) alterations than by KCNQ1 variants. The study was approved by the ethical committee of the Medical Faculty of the RWTH Aachen University (EK303-18).

\section{CNV analysis of the KCNQ1 gene}

The patient's DNA samples were analysed for deletions or duplications of the KCNQ1 gene by MLPA (assay P114-B3-0517, MRC-Holland) following the manufacturer's instructions.

\section{Variant detection by amplicon-based next generation sequencing}

All patients were screened for SNVs in KCNQ1 by an amplicon-based next generation sequencing approach. First, the exons of the whole coding region of KCNQ1 were amplified by polymerase chain reaction (PCR); primers and PCR conditions are listed in supplementary tables. The 17 PCR assays were multiplexed; finally, three PCRs were performed per sample. For the amplification of exon 1, a nested PCR assay was used (see supplementary tables). PCR products of the same individual were pooled, and $3^{\prime}-\mathrm{A}$ overhangs were added to the PCR products by using the NEBNext Ultra II End Prep Enzyme Mix (New England Biolabs, Ipswich, USA) to allow the binding of the specifically designed oligo adapters (see supplementary tables). The adapters are based on the sequences of the transposase oligos published in Bogdanoff et al. [24] (Fig. 3). These oligos were annealed and phosphorylated using the T4 Polynucleotide Kinase (New England Biolabs) (see supplementary tables) and subsequently ligated to the PCR products, using the Blunt/TA Ligase Master Mix (New England Biolabs) (see supplementary tables). The ligated oligo adapters serve as connectors between the PCR product and the Nextera index primers (Illumina, San Diego, USA). To add the sequencing adapters and to index the samples, a second PCR with the pooled PCR products and the Illumina Nextera Index Adapter N70X and E50X was performed using the KAPA Hifi Ready Mix (Roche Sequencing and Life Science, Kapa Biosystems, Wilmington, USA) (see supplementary tables). The concentration of the final library was determined by quantitative PCR and sequenced on a MiSeq Nano Flowcell (Illumina, San Diego, USA) with $2 \times 150$ cycles. FastQ files were generated by the built-in standard MiSeq pipeline. Data analysis and variant calling were performed with SeqNext (JSI medical systems, Ettenheim, Germany). Synonymous variants and variants with a frequency $>1 \%$ in public databases were discarded. Variant confirmation and segregational analyses were conducted by Sanger sequencing.

\section{Supplementary information}

Supplementary information accompanies this paper at https://doi.org/10. 1186/s13148-020-00856-y.

Additional file 1: Supplementary tables. Primer sequences and PCR conditions for the amplicon-based NGS analysis of KCNQ1.

\section{Abbreviations}

BWS: Beckwith-Wiedemann syndrome; CNV: Copy number variant; ECG: Electrocardiogram; GOM: Gain of methylation; IC1: Imprinting Centre 1 in 11p15.5; IC2: Imprinting Centre 2 in 11p15.5; LOM: Loss of methylation; LQTS: Long-QT syndrome; MLID: Multilocus imprinting disturbances; MLPA: Multiplex ligation-dependent probe amplification; MSMLPA: Methylation-specific multiplex ligation-dependent probe amplification; NGS: Next generation sequencing; SNV: Single nucleotide variant

\section{Acknowledgements}

Not applicable

\section{Authors' contributions}

CE analysed the DNA samples by MLPA and amplicon-based NGS and was a major contributor in writing the manuscript. MB designed the ampliconbased NGS approach. SK and UM provided the clinical data of the patient. TE revised the manuscript and coordinated the trials. All authors read and approved the final manuscript.

\section{Funding}

This work was funded by the Deutsche Forschungsgemeinschaft (DFG, EG110/15-1; 948/32-1 FUGG).

\section{Availability of data and materials}

The datasets generated and/or analysed during the current study are not publicly available due to privacy restrictions but are available from the corresponding author on reasonable request.

Ethics approval and consent to participate

The study was approved by the ethical committee of the Medical Faculty of the RWTH Aachen University (EK303-18).

Consent for publication

Written informed consent for publication of their clinical details was obtained from the parent of the patient. A copy of the consent form is available for review by the Editor of this journal.

\section{Competing interests}

The authors declare that they have no competing interests.

\section{Author details}

${ }^{1}$ Institute of Human Genetics, Medical Faculty, RWTH Aachen University, Pauwelsstr. 30, 52074 Aachen, Germany. 'University Children's Hospital, Heidelberg University, Heidelberg, Germany. ${ }^{3}$ Institute of Human Genetics, Heidelberg University, Heidelberg, Germany. ${ }^{4}$ Il. Department of Paediatrics, Semmelweis University, Budapest, Hungary.

Received: 10 March 2020 Accepted: 23 April 2020

Published online: 11 May 2020

\section{References}

1. Beckwith JB. Extreme cytomegaly of the adrenal fetal cortex, omphalocele, hyperplasia of kidneys and pancreas, and Leydig-cell hyperplasia: another syndrome? Western Soc Pediatr Res. 1963. 
2. Wiedemann HR. Familial malformation complex with umbilical hernia and macroglossia--a "new syndrome". J Genet Hum. 1964;13:223-32.

3. Brioude F, Kalish JM, Mussa A, Foster AC, Bliek J, Ferrero GB, et al. Expert consensus document: Clinical and molecular diagnosis, screening and management of Beckwith-Wiedemann syndrome: an international consensus statement. Nature reviews Endocrinology. 2018;14(4):229-49.

4. Weksberg R, Shuman C, Smith AC. Beckwith-Wiedemann syndrome. Am J Med Genet C Semin Med Genet. 2005;137C(1):12-23.

5. Choufani S, Shuman C, Weksberg R. Molecular findings in BeckwithWiedemann syndrome. Am J Med Genet C Semin Med Genet. 2013;163C(2): $131-40$.

6. Eggermann T, Algar E, Lapunzina P, Mackay D, Maher ER, Mannens M, et al. Clinical utility gene card for: Beckwith-Wiedemann Syndrome. Eur J Hum Genet. 2014;22(3). https://doi.org/10.1038/ejhg.2013.132.

7. Heide S, Chantot-Bastaraud S, Keren B, Harbison MD, Azzi S, Rossignol S, et al. Chromosomal rearrangements in the 11 p15 imprinted region: 17 new 11 p15.5 duplications with associated phenotypes and putative functional consequences. J Med Genet. 2018;55(3):205-13.

8. Brioude F, Lacoste A, Netchine I, Vazquez MP, Auber F, Audry G, et al. Beckwith-Wiedemann syndrome: growth pattern and tumor risk according to molecular mechanism, and guidelines for tumor surveillance. Horm Res Paediatr. 2013;80(6):457-65.

9. Lam WW, Hatada I, Ohishi S, Mukai T, Joyce JA, Cole TR, et al. Analysis of germline CDKN1C (p57KIP2) mutations in familial and sporadic BeckwithWiedemann syndrome (BWS) provides a novel genotype-phenotype correlation. J Med Genet. 1999;36(7):518-23.

10. Niemitz EL, DeBaun MR, Fallon J, Murakami K, Kugoh H, Oshimura M, et al. Microdeletion of LIT1 in familial Beckwith-Wiedemann syndrome. Am J Hum Genet. 2004;75(5):844-9.

11. Zollino M, Orteschi D, Marangi G, De Crescenzo A, Pecile V, Riccio A, et al. A case of Beckwith-Wiedemann syndrome caused by a cryptic 11 p15 deletion encompassing the centromeric imprinted domain of the BWS locus. J Med Genet. 2010;47(6):429-32

12. Algar E, Dagar V, Sebaj M, Pachter N. An 11p15 imprinting centre region 2 deletion in a family with Beckwith Wiedemann syndrome provides insights into imprinting control at CDKN1C. PLoS One. 2011;6(12):e29034.

13. Baskin B, Choufani S, Chen YA, Shuman C, Parkinson N, Lemyre E, et al. High frequency of copy number variations (CNVs) in the chromosome 11p15 region in patients with Beckwith-Wiedemann syndrome. Hum Genet. 2014; 133(3):321-30

14. Beygo J, Joksic I, Strom TM, Ludecke HJ, Kolarova J, Siebert R, et al. A maternal deletion upstream of the imprint control region 2 in 11 p15 causes loss of methylation and familial Beckwith-Wiedemann syndrome. Eur J Hum Genet. 2016;24(9):1280-6.

15. Demars J, Rossignol S, Netchine I, Lee KS, Shmela M, Faivre L, et al. New insights into the pathogenesis of Beckwith-Wiedemann and Silver-Russell syndromes: contribution of small copy number variations to 11 p15 imprinting defects. Hum Mutat. 2011;32(10):1171-82.

16. Beygo J, Burger J, Strom TM, Kaya S, Buiting K. Disruption of KCNQ1 prevents methylation of the ICR2 and supports the hypothesis that its transcription is necessary for imprint establishment. Eur J Hum Genet. 2019.

17. Singh VB, Sribenja S, Wilson KE, Attwood KM, Hillman JC, Pathak S, et al. Blocked transcription through KvDMR1 results in absence of methylation and gene silencing resembling Beckwith-Wiedemann syndrome. Development. 2017:144(10):1820-30.

18. Kapplinger JD, Tester DJ, Salisbury BA, Carr JL, Harris-Kerr C, Pollevick GD, et al. Spectrum and prevalence of mutations from the first 2,500 consecutive unrelated patients referred for the FAMILION long QT syndrome genetic test. Heart Rhythm. 2009;6(9):1297-303.

19. Crotti L, Celano G, Dagradi F, Schwartz PJ. Congenital long QT syndrome. Orphanet J Rare Dis. 2008;3:18.

20. Napolitano C, Priori SG, Schwartz PJ, Bloise R, Ronchetti E, Nastoli J, et al. Genetic testing in the long QT syndrome: development and validation of an efficient approach to genotyping in clinical practice. JAMA. 2005;294(23): 2975-80.

21. Valente FM, Sparago A, Freschi A, Hill-Harfe K, Maas SM, Frints SGM, et al. Transcription alterations of KCNQ1 associated with imprinted methylation defects in the Beckwith-Wiedemann locus. Genet Med. 2019.

22. Gurrieri F, Zollino M, Oliva A, Pascali V, Orteschi D, Pietrobono R, et al. Mild Beckwith-Wiedemann and severe long-QT syndrome due to deletion of the imprinting center 2 on chromosome 11p. Eur J Hum Genet. 2013;21(9):965-9.
23. Kaltenbach S, Capri Y, Rossignol S, Denjoy I, Soudee S, Aboura A, et al. Beckwith-Wiedemann syndrome and long QT syndrome due to familialbalanced translocation $\mathrm{t}(11 ; 17)(\mathrm{p} 15.5 ; \mathrm{q} 21.3)$ involving the KCNQ1 gene. Clin Genet. 2013;84(1):78-81.

24. Bogdanoff $D$, Jou T, Lee B. Characterization of action and efficiency of Tn5 transposase through comparative qPCR. J Exp Microbiol Immunol (JEMI). 2015.

\section{Publisher's Note}

Springer Nature remains neutral with regard to jurisdictional claims in published maps and institutional affiliations.
Ready to submit your research? Choose BMC and benefit from:

- fast, convenient online submission

- thorough peer review by experienced researchers in your field

- rapid publication on acceptance

- support for research data, including large and complex data types

- gold Open Access which fosters wider collaboration and increased citations

- maximum visibility for your research: over $100 \mathrm{M}$ website views per year

At BMC, research is always in progress.

Learn more biomedcentral.com/submissions 